\title{
LA ESCUELA DE PENSAMIENTO HUMANBECOMING: UNA ALTERNATIVA PARA LA PRÁCTICA DE LA ENFERMERÍA ${ }^{1}$
}

\author{
THE HUMANBECOMING SCHOOL OF THOUGHT: \\ AN ALTERNATIVE TO THE PRACTICE OF NURSING
}

\author{
Vivian VÍlChEZ-Barboza V. \\ Tatiana Paravic-Klijn T. ${ }^{* *}$ \\ Alide Salazar Molina ${ }^{* * *}$
}

\begin{abstract}
RESUMEN
El presente artículo tiene por objetivo exponer de forma general la visión de la Escuela del pensamiento $H u$ manbecoming de Parse, como una alternativa para guiar la práctica de la Enfermería, evidenciándose a través de las investigaciones que se han desarrollado con esta teoría en el ejercicio profesional. Para ello se enfatiza en los principios, conceptos, paradojas y postulados de la Teoría Humanbecoming, la cual se centra en el accionar del ser humano, su calidad de vida y salud, a partir de sus contextos, vivencias y creencias. Dichos fundamentos teóricos son desarrollados a partir de la filosofía de la ciencia humana y del existencialismo, los cuales se fusionan y entregan un innovador esquema para la disciplina de Enfermería. Además se aborda la propuesta que hace esta Escuela a los y las profesionales de Enfermería para vivenciar el Humanbecoming, a partir de las herramientas que otorga para guiar la práctica profesional.
\end{abstract}

Palabras clave: Teoría de Enfermería, atención de enfermería, enfermería.

\begin{abstract}
This article aims to expose the vision of the School of Parse Humanbecoming School of Thought, as an alternative to guide nursing practice, thus evidence by the research that has been developed with this theory in practice. For this, the principles, concepts, paradoxes and postulates of the Humanbecoming theory are enphasized, which focuses on the actions of human beings, their quality of life and health, from their contexts, experiences and beliefs. These theoretical foundations are developed from the philosophy of human science and existentialism; both philosophies deliver an innovative work plan for the nursing discipline. It also addresses this school proposal to the nursing professionals to experience the Humanbecoming, from the tools granted to guide professional practice.
\end{abstract}

Key words: Nursing theory, nursing care, nursing.

Fecha recepción: 15/01/13 Fecha aceptación: 09/07/13

\footnotetext{
${ }^{1}$ Fuente de Apoyo Económico: CONICYT-PCHA/EVENTOS Y CURSOS CORTOS EN CHILE Y AL EXTRANJERO 201281120482, para asistencia a la15 ${ }^{\text {th }}$ Bienal Internacional Conferencia en Humanbecoming. Canadá, 2012.

* Enfermera. Alumna Programa Doctorado en Enfermería, Departamento de Enfermería, Facultad de Medicina, Universidad de Concepción, Chile. Email: vivian.vilchez@ucr.ac.cr

${ }^{* *}$ Enfermera. Profesora Departamento de Enfermería, Facultad de Medicina, Universidad de Concepción, Chile. Email: tparavic@udec.cl

${ }_{* * *}$ Enfermera-Matrona. Profesora Departamento de Enfermería. Facultad de Medicina. Universidad de Concepción. Email: alisalaz@udec.cl
} 


\section{INTRODUCCIÓN}

La Enfermería puede contribuir al sistema de salud con alternativas para la resolución de necesidades impuestas por los fenómenos contemporáneos, propiciando innovación en la atención en los diferentes escenarios laborales por medio de la construcción de conocimiento.

En este sentido, el uso de las teorías y modelos de Enfermería permite el acercamiento de la teoría a la práctica, dándole así un sustento al "hacer" al proveerlo de un marco propio para interpretar los hallazgos y guiar la práctica, la investigación, la educación y la gerencia; mostrando una apropiación clara en el "ser" y el "saber". Ello otorga una relevancia social, profesional y científica a la Enfermería, esto es lo que se conoce como "Sistema de Conocimiento en Enfermería Conceptual-Teórico-Empírico" (sistema CT-E) (1), definido como el servicio que Enfermería brinda a la sociedad guiado por el conocimiento específico, al articularse con las teorías de esta disciplina, lo que permite una interacción fluida y competitiva con las demás disciplinas.

En concordancia con lo anterior, este artículo expone la visión de la Teoría Humanbecoming, la cual fue evolucionando hasta convertirse en una Escuela de Pensamiento, que presenta una alternativa para guiar la práctica de la Enfermería, evidenciándose a través de las investigaciones que se han desarrollado con esta teoría en el ejercicio profesional.

\section{Antecedentes de la Teoría Humanbecoming}

La teoría planteada por Rosemarie Rizzo Parse emerge a principios de los años 80 (Figura 1), en el contexto anglosajón, específicamente en la Universidad de Duquesne en Pittburburg, Estados Unidos. Nace como respuesta desde la Enfermería al movimien- to global que apelaba al individualismo y a la reducción de servicios de salud por la recesión económica (2). Propone un ejercicio disciplinar desde un paradigma de simultaneidad o transformación, que brinda una alternativa a los paradigmas imperantes: positivista (salud como ausencia de enfermedad) y de integración (salud como algo biopsico-social-espiritual) (3). En la actualidad, nombra al paradigma que guía a esta teoría Humanuniverse (4).

Esta teoría fue publicada por primera vez con el nombre Hombre-Vida-Salud: Una Teoría de Enfermería (Man-Living-Health: A Theory of Nursing) (5), enmarcada como ciencia humana, fundamentada en lo propuesto por Dilthey y Gadamer. Además toma los principios de la filosofía existencialista de Heidegger, Sartre y Mearleau-Ponty (6) y desde la Enfermería toma como referencia la Teoría del Ser Humano Unificado de Rogers. Es de este modo que esta teoría define su ciencia y su arte centrada en la vivencia de experiencias universales: vivir con expectativas de cambio, esperanza, miedo, alegría, satisfacción, seguridad, sufrimiento, entre otros; como fenómenos de la calidad de vida y salud (7).

Un ejemplo de la investigación que ha abordado los fenómenos universales, es el estudio que exploró el significado de las expectativas de cambio en personas que habían experimentado la cancelación inesperada de una cirugía a la que iban a ser sometidos; los resultados mostraron que las personas que atraviesan esta situación sufren un desencanto e incertidumbre, además se sienten humilladas y escépticas acerca de lo que va a suceder; por lo tanto, sus expectativas de cambio van dirigidas a visualizar posibles alternativas para reconciliarse con los sentimientos de desencanto, incertidumbre, humillación y escepticismo (8).

A través del tiempo la teoría ha mantenido su esencia, aunque su autora ha realizado modificaciones que corresponden a la profundización de su estudio en la fundamen- 
tación y evolución filosófica de la misma. Por esta razón, es que en 1992 la teoría fue renombrada Teoría de la Evolución Humana o del Desarrollo Humano (The Human Becoming Theory) (5).

En 1998 Rosemarie Rizzo Parse identifica Human Becoming como una escuela de pensamiento, por haberse convertido en un punto de vista teórico que tiene una comunidad de estudiosos de la teoría, que expone ideas innovadoras y visionarias acerca de la Enfermería y la forma de brindar atención (6). Direcciona una ontología compuesta por supuestos, postulados y principios con conceptos y paradojas, que se basan en la interrelación ser humano-entorno; una epistemología que focaliza en la investigación formal de los fenómenos universales de salud y calidad de vida que son vividos por las personas en diferentes momentos de la vida, con el fin de conceptualizarlos y contribuir a la práctica de la Enfermería, para lo cual cuenta con tres metodologías que buscan la forma de acercar la investigación a la práctica.

Posteriormente, en el 2007, producto del agitado contexto de la sociedad actual, del gran desarrollo tecnológico y de la importancia del costo-efectividad en el sistema de salud, Parse reforma su teoría clarificando los principios y enfatizando en la idea de co-creación, lo que le permitió realizar un nuevo cambio al nombre de la teoría: acopló las palabras ser humano (human) y evolución (becoming) y lo volvió indivisible Humanbecoming (9), respondiendo a una total congruencia con su fundamentación filosófica.

Figura 1. Evolución Histórica de la Teoría Humanbecoming.

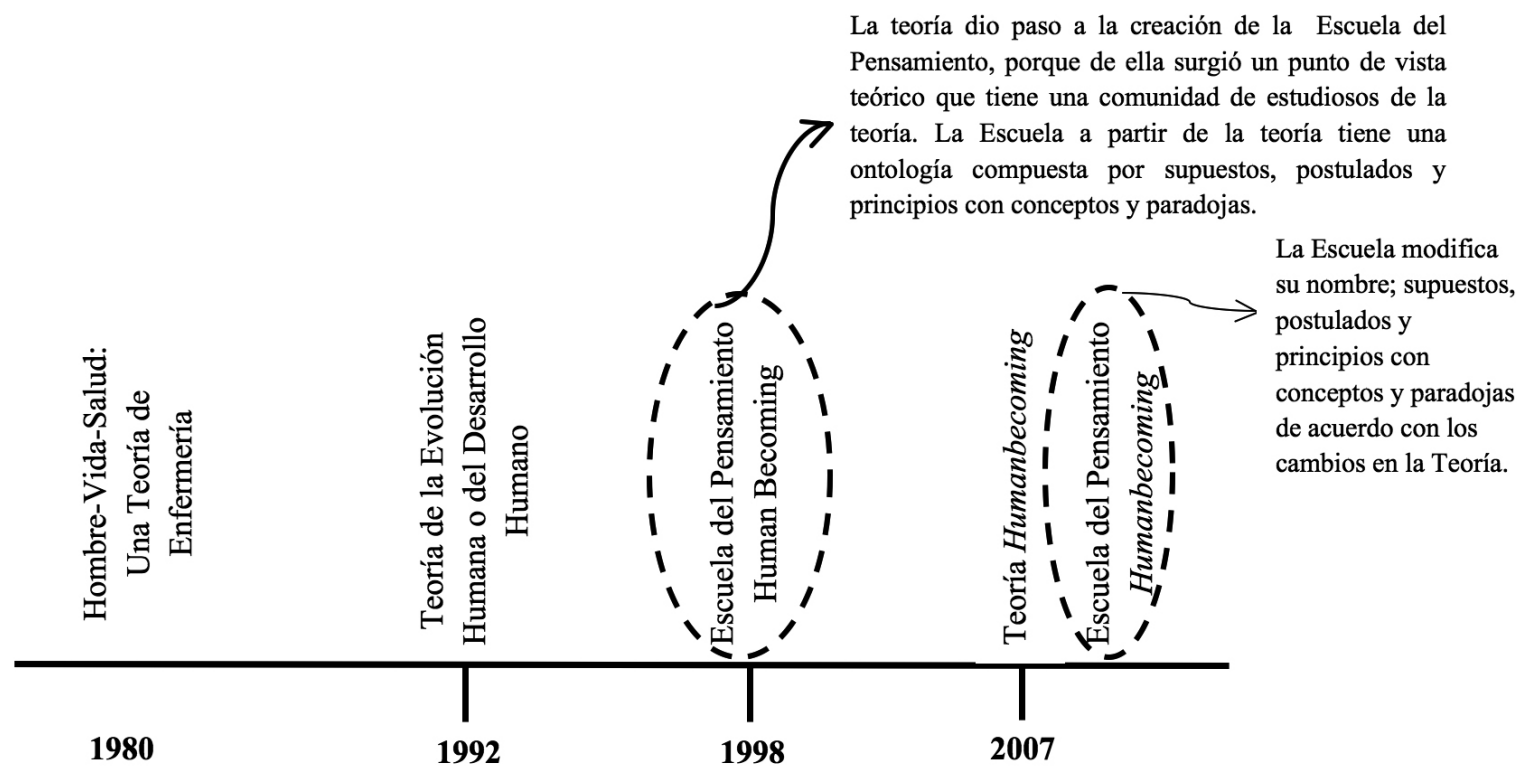

Fuente: Elaboración autoras

La comprensión de esta visión requiere de una mentalidad dispuesta a reconocer que el ser humano no puede ser reducido a la suma de sus partes bio-psico-social-espiritual; pues el ser humano se caracteriza por tener patrones de relación con ellos mismos, con otros y el universo; otorgándole un papel activo y co-participativo en la vivencia de la salud, con la total potestad para elegir cómo vivirla (4). Por lo tanto, desde este enfoque el 
ser, hacer y saber del profesional de Enfermería tiene un fin bien definido, distinto al tradicional, ya que se centra en la relación con el ser humano y fija como meta profesional el logro de vivir con calidad y salud (4), es decir, con bienestar, desde la perspectiva que posee el individuo y su familia -entendiendo familia como las personas con las que se tiene una relación estrecha- (10).

\section{Escuela del Pensamiento Humanbecoming}

La Escuela del Pensamiento Humanbecoming es un sistema específico de pensamiento acerca del universo humano ( $h u$ manuniverse) y la Teoría Humanbecoming (11). Este pensamiento se lleva a la práctica a través de los modelos desarrollados: enseñanza-aprendizaje (teaching-learning) (5), liderazgo-permanente (leading-follow) (12) y comunidad de cambio (community change) (11). También por medio de los métodos de investigación: Método de Estudio Parse, Método Hermenéutico Human Becoming y Cualitativo Descriptivo Pre-proyecto-Proceso-Postproyecto (13).

Esta Escuela toma los conceptos de los metaparadigmas de la Teoría Humanbecoming: Enfermería, ciencia que brinda un servicio a la humanidad, tiene como razón principal al ser humano en interrelación directa con el entorno, la calidad de vida y la salud (14). Salud, proceso evolutivo que surge a partir de las experiencias vividas, además involucra la calidad de vida desde la conceptualización individual de cada grupo o persona, de acuerdo a sus valores y modos de vida. Persona, ser humano que convive con una serie de miradas diferentes, que le permiten ver el mundo y explicar las situaciones que se presentan en su contexto. Ante esto, la persona es un ser con apertura para el conocimiento, que va creando sus propias percepciones, a partir de las cuales toma decisiones y desarrolla capacidades de elección. Entorno, la interrelación ser humano-entorno está mediada por procesos relacionados con costumbres, relaciones interpersonales, valores, contacto con los diferentes contextos (familiar, laboral, social, entre otros) y los fenómenos que se estructuran a partir de la experiencia de vida (miedo, alegría, esperanza, paz, seguridad, expectativas de cambio, satisfacción, entre otras) $(15,16)$. Por lo tanto, el ser humano y el entorno son inseparables.

Esta Escuela representa sus supuestos provenientes de la Teoría Humanbecoming, por medio del siguiente símbolo (5):

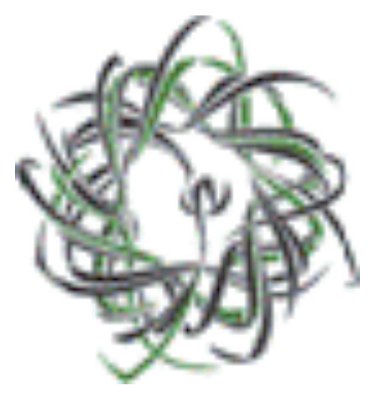

Los colores blanco y negro significan los opuestos o paradojas que tiene el ser humano. El color verde simboliza la esperanza. La unión en el centro significa el proceso de co-creación enfermera(o)-persona y los remolinos verdes y negros entremezclando se refieren al proceso continuo de evolución del ser humano-universo.

Los cuatro supuestos, descritos a continuación, son afirmaciones filosóficas que reflejan la forma de conceptualizar las creencias acerca del ser humano, el universo y la salud planteadas por la Teoría Humanbecoming (17). Para contribuir a su comprensión se ilustrarán con la aplicación de estos postulados en la práctica de la Enfermería, tomando como referencia una investigación realizada en España en personas con Alzheimer (18).

1. Humanbecoming es estructurar significados y tomar decisiones en las situaciones de salud.

El primer supuesto se aplica cuando una persona acude al sistema de salud a consultar sobre su situación y, por lo general, se le solicita modificar su estilo de vida y desde esta 
teoría se establece lo que los autores llaman un "pacto-compromiso" entre la persona usuaria y el profesional de Enfermería; este "pacto-compromiso" debe incorporar lo que significa para la persona implementar esas modificaciones recomendadas, con el fin de tomar decisiones basadas en su realidad, con el fin de que la/el Enfermera/o no se sienta frustrada/o en cuanto a los resultados que obtiene (18).

\section{Humanbecoming es configurar los patro-} nes rítmicos de relación con el universo humano (humanuniverse).

Se destaca cuando las/os profesionales de Enfermería ayudan a las personas usuarias al desarrollo de sus posibilidades para que encuentren sus propias soluciones, sobre todo cuando se trabaja con personas con enfermedades crónicas. La configuración de los patrones rítmicos se relaciona con el acompañamiento que brinda el/la enfermero/a en las diferentes emociones por las que transita la persona usuaria: alegrías, desánimo, entre otros (18).

\section{Humanbecoming es co-trascender ilimita-} damente con las posibilidades que emergen.

Se refiere a la inclusión en el plan de cuidados de las esperanzas y los proyectos de la persona con la que se trabaja (18).

\section{Humanbecoming es co-crear en el universo} humano (humanuniverse) una sinfonía perfecta.

Se ejecuta este supuesto cuando la/el enfermera/o colabora con las personas a entender, imaginar y/o sentir, lo que les implica modificar sus patrones de salud, cambiando la prioridad de sus valores (18). Por ejemplo, en el estudio donde participaron personas con Alzheimer, uno de los señores manifestó que tenía dos valores prioritarios en la etapa de la enfermedad en la que se encontraba: la seguridad y la necesidad de tranquilidad en la convivencia con su mujer (18).

Por otra parte, la Teoría le otorga a la Es- cuela del Pensamiento cuatro postulados, con el fin de promover la comprensión del desarrollo humano (5):

a) Infinito: el conocimiento en el ser humano es indivisible e ilimitado, se forma a partir de las experiencias de vida, las cuales involucran desarrollo personal (5). Es por ello que cuando se diagnostica a una persona con una enfermedad determinada, es importante explorar qué conocimiento tiene sobre esta condición de salud. El estudio realizado en profesionales de Enfermería de la atención primaria en Brasil hace referencia a que la estrategia de atención de las personas con hipertensión arterial debe estar encaminada no sólo a la enfermedad, sino también al aspecto emocional y físico, y que la persona exprese los cambios que ha experimentado desde el diagnóstico (19).

b) Paradoja: se entiende paradoja como una figura retórica que consiste en la unión de dos ideas que, aparentemente, en un principio parecen imposibles de concordar (20). Son vivencias rítmicas. Es decir, en el desarrollo humano hay momentos donde existe conexión con otros y el universo, mientras que al mismo tiempo puede existir separación y soledad (5). Por ejemplo, cuando sucede un acontecimiento que causa sufrimiento, las personas a menudo no encuentran las palabras para describir la situación por la que está atravesando y la experiencia que significa en su vida. Esta inexpresión puede promover la incomunicación y distanciarse física y socialmente de otros por la aflicción (21). Lo anterior se evidencia en los resultados obtenidos en la investigación realizada en mujeres con embarazo de alto riesgo, las cuales tienen como expectativa de cambio dentro de la atención de Enfermería que reciben, nutrir sus redes de apoyo para establecer vínculos saludables, ya que la situación que viven les genera mucha an- 
gustia y sentimientos encontrados, lo cual provoca que sus relaciones interpersonales en ocasiones se afecten (22).

c) Libertad: es contextualmente construir liberación. La elección de seguir adelante dejando de "cargar" con asuntos que no son nuestra responsabilidad se convierte en liberación personal. Este postulado se ilustra en el caso de las mujeres que son abuelas y dedican todo su tiempo para cuidar a su nieto/a, con lo cual no les queda espacio para desarrollar otro tipo de actividades, lo que las limita en el desarrollo personal de otras capacidades en esta etapa de su vida.

d) Misterio: es lo inexplicable, lo que no es completamente conocido en la vida y va ligado con la evolución humana. Por ejemplo, los problemas de investigación que surgen de esta teoría: ¿cuáles son las experiencias que vive cotidianamente el individuo en sufrimiento psíquico y cuáles son los significados de estas experiencias? (23), ¿qué significa ser un cuidador/a familiar de una persona con trastorno mental? (24), ¿qué es calidad de vida en una persona con Alzheimer? (18), entre otros.

De los cuatro supuestos y las cuatro paradojas emergen tres temas: significado, ritmicidad y trascendencia. Cada tema conduce a un principio y cada principio es específico con tres conceptos y sus paradojas $(5,7)$.

\section{Principio 1: Estructurando significados.} Trata de dar respuesta a la interrogante ¿cómo los seres humanos estructuran el significado de su realidad? La respuesta se divide en dos: proyección de la imagen y el valor del lenguaje $(5,7)$.

Los significados son una realidad personal que continuamente se estructuran y son diferentes en el pasado y en el presente, lo que incide en el futuro $(5,7)$. El ser huma- no co-crea la realidad a través de la conexión con las personas, tecnología y con diversas formas de arte. Es la manera en que el ser humano vive su realidad.

Por ejemplo, en la adolescencia la búsqueda de la identidad conlleva a un cambio importante en la forma de vida, surgen cuestionamientos sobre ¿Quién soy? ¿Qué quiero? ¿Hacia dónde voy? y hay un sentimiento de inmortalidad que acompaña esta etapa y hace que la medida de los riesgos sea alterada. Junto con esto la aceptación en un grupo es fundamental y la opinión que emitan los pares se convierte en indispensable, lo que incide en las formas de comunicación. En cambio en la adultez la visión de mundo es totalmente diferente, ya se tienen claros los cuestionamientos y existen variantes en la importancia de la opinión que emiten los pares y en la forma de comunicación. Estos son aspectos que el/la profesional de Enfermería debe considerar al gestionar el cuidado en los diferentes grupos poblacionales. Es necesario conocer cómo estructuran las personas usuarias el significado de su realidad, porque esto es lo que les da el propósito de vida y la posibilidad de explorar alternativas de cambio $(5,7)$. Por lo tanto, se requiere comprender algunos conceptos como; imagen, valoración y lenguaje, con sus respectivas paradojas; ya que en conjunto son los que forman la estructura del significado.

a) Imagen: es mostrar la realidad personal a través del conocimiento explícito-tácito, esto es hacer real las ideas o eventos (25). La paradoja de este concepto es: explícitotácito. Esto significa poder expresar con palabras lo que es conocido, pero también lo que no es conocido. La imagen de la realidad personal se extiende al lenguaje y la valoración (25). Esto se ejemplifica cuando una persona ingresa a un servicio y la/ el enfermera/o que lo recibe, junto con realizar los procedimientos requeridos, se interesa por explorar los sentimientos y pensamientos. Cuando la/el enfermera/o 
logra un proceso de apertura en la persona usuaria, ésta puede descubrir nuevos aspectos de sí misma en ese momento: ¿Por qué reacciona de ese modo? ¿Cuál es su mayor preocupación? Lo cual no es explícitamente conocido anteriormente.

b) Valoración: es el proceso de elegir y vivir con las creencias que apreciamos. La paradoja de valorar es: confirmar-no confirmar (5). Es decir, en ocasiones se elige de manera simultánea de acuerdo a las creencias y otras veces en contra de las creencias. Las creencias que más se aprecian se hacen realidad a través de la imagen que se proyecta y son expresadas a través del lenguaje (26). Se ejemplifica en las investigaciones sobre las experiencias de vida de los fenómenos universales, al mostrar la valoración que realizan las personas participantes en determinadas situaciones de salud. Un ejemplo lo constituye el estudio que abordó la experiencia de vivir el sufrimiento en la población general, uno de los conceptos resultantes como principal en la definición del sufrimiento representa la valoración realizada; sufrimiento como una angustia paralizante que permite mirar preciosas posibilidades, donde se integran en la estructura de este fenómeno la impotencia, la desdicha y la mirada hacia las cosas preciadas (27).

c) Lenguaje: expresa el significado a través de hablar-estar en silencio (lenguaje verbal), de moverse-estar quieto (lenguaje no verbal). Como las personas se interrelacionan con otros y el universo, los ritmos paradójicos de hablar-estar en silencio y moverse-estar quieto simultáneamente vislumbran una realidad personal de los valores que rigen a cada individuo (5). Por ejemplo, una investigación brasileña reflexionó sobre las experiencias vividas en el cotidiano de un individuo en sufrimiento psíquico, identificando por medio del lenguaje los significados de estas ex- periencias. Los significados encontrados fueron: conviviendo con los recuerdos de la infancia y la co-construcción del sufrimiento psíquico. El primer significado permitió al profesional de Enfermería conocer las dificultades de sus primeras experiencias con la enfermedad, cómo constituyen sus relaciones con los otros, lo cual influye en la construcción de su identidad y va a determinar la forma de trabajar su autoestima y autoimagen. El segundo significado, la co-construcción del sufrimiento psíquico, evidencia la necesidad de considerar la exclusión social del individuo con este tipo de padecimiento, el sufrimiento y alteración que se produce en el ambiente familiar, en los planes de atención (23).

\section{Principio 2: Configuración de patrones} rítmicos. Se refiere a las paradojas de las experiencias humanas $(5,9)$. Su función es invitar a la reflexión. Los ritmos paradójicos son revelación-ocultación, permitir-limitar y conexión-separación.

a) Revelación-ocultación: está unida a la paradoja desenmascarar-enmascarar. Siempre hay más que decir y más que conocer de nosotros mismos y sobre los demás. A veces transmitimos mensajes muy claros y otras veces no. Algunos aspectos de la realidad y la experiencia se han ocultado (7). Esto es contar o no una historia, es exponer una situación mientras al mismo tiempo la ocultamos. Por ejemplo: las familias de personas con enfermedad psíquica inicialmente niegan o esconden el diagnóstico de enfermedad mental; en la medida que la familia se siente esclarecida, comienza a entender la dolencia y diseñar estrategias de afrontamiento (24).

b) Permitir-limitar: está unida a la paradoja potenciar-restringuir. Esto es acerca de abrir y cerrar puertas al hacer elecciones en la vida (5). Cuando una puerta se abre 
otras se cierran y viceversa. Cada elección que se hace significa infinitas posibilidades como también limitaciones. No se pueden realizar todas las posibilidades a la vez. Un ejemplo lo constituye el estudio de caso sobre un individuo con deficiencia visual que es diagnosticado con Síndrome de Inmunodeficiencia Adquirida (SIDA) (28). El diagnóstico desestabilizó el equilibrio personal y familiar, al no encontrarse preparados para enfrentar la situación. Este hombre era autovalente y después del diagnóstico sufrió drásticos cambios en su manera de vivir, haciéndose necesario reestructurar todo el medio donde vivía, por lo que fue fundamental dirigir las acciones de Enfermería a que él asumiera su situación y a que la familia lo incentivara y le ofreciera motivos para un buen vivir dentro de su situación (28).

c) Conexión-separación: está unida a la paradoja atender-distanciarse. Este es el proceso de moverse en una dirección y alejarse de otras. Formas en que las personas crean pautas para conectarse y separarse de la gente, de los proyectos o de las situaciones (6). Se ilustra en una investigación que desarrolla la temática sentir inseguridad como una experiencia universal de vida, en personas con enfermedad cardiaca congestiva crónica y reporta como hallazgo central que dicho fenómeno para estas personas es una perturbación que genera disconfort, es como estar vigilante para anticiparse a las posibilidades con las que cuentan; destacan que en los momentos donde más requieren tolerancia surgen valiosas alianzas (29). La/el enfermera/o debe fomentar esas alianzas, para lo cual debe conocer el proyecto de vida de las personas usuarias, el cual incluye metas, recursos y redes de apoyo con los que cuenta.

Principio 3. Co-trascendencia. Es movilizarse más allá, con las posibilidades que sur- gen, lo cual permite co-crear con otros y el universo (5). Movilizarse más allá, significa atreverse a tomar decisiones entre un sinnúmero de posibilidades y esas decisiones involucran cambios en la vida. Esto ocurre porque el ser humano no es estático, siempre está en un proceso de evolución, por lo que las posibilidades de cambio se presentan a diario. Son las maneras infinitas que existen para ser y evolucionar dentro de las situaciones. Cuando se elige, las posibilidades son actualizadas y emergen nuevas, entre las cuales uno elige otra vez (30). Los conceptos que desarrolla este principio son:

a) Poder: Se refiere a la fuerza y energía que impulsa al ser humano a vivir. Este concepto esta conectado con las paradojas: emprender-resistir, comprobar-no comprobar y ser-no ser (5). El poder transmite el significado de lucha y perseverancia en la vida, es lo que otorga la voluntad de continuar a pesar de las dificultades que se enfrenten. Comprende la capacidad de mirar el conflicto como la oportunidad de aclarar el significado y los valores, así como una manera de generar esperanzas y sueños. Las/los enfermeras/os pueden potenciar el poder cuando acompañan a las personas usuarias en el proceso de mirar el conflicto como una oportunidad para realizar cambios favorables en su calidad de vida, los cuales le brinden mayor bienestar (7). Pero también el poder se evidencia en el afrontamiento que realizan los profesionales de Enfermería ante las adversidades que presentan los sistemas de salud, como lo muestra un estudio, al señalar que trabajar en el área de salud constituye un gran desafío profesional, por la gran cantidad de demandas de parte de los usuarios/as hacia el servicio de salud versus las limitaciones financieras, de infraestructura y de recurso humano y la disyuntiva entre cantidad y/o calidad de atención (19). 
b) Origen: es la creación personal, que conecta con personas y proyectos y da la posibilidad de movilizarse más allá de lo establecido. Las paradojas son adaptaciónno adaptación (las personas buscan ser como otros y diferentes) y certeza-incertidumbre (reinventarse). Ambos son resultado de las elecciones que se toman en la vida $(5,30)$. Las/los enfermeras/os son testigos de cómo las personas se reinventan cuando tienen que tomar decisiones ¿cómo cambian las personas sus patrones de salud? ¿Qué acciones van a tomar y por qué? Los diferentes grupos de apoyo que se establecen en los Centros de Salud son ejemplos de cómo las personas se reinventan y viven las paradojas. Un caso lo representan los grupos dirigidos a las personas adultas mayores que tienen como objetivo estimular las habilidades cognitivas y psicomotoras, sin embargo, para los y las participantes se convierten en una oportunidad de establecer relaciones de amistad, salir de paseo, vivir nuevas experiencias, compartir sentimientos y realidades de vida similares, pero a la vez les permite tener independencia y motivación.

c) Transformación: es el proceso de trascendencia, lo cual indica que la persona se esforzó para tener claridad del rumbo que desea dar a su vida, para lo cual logra tomar decisiones de forma segura y con disposición para asumir las consecuencias tanto positivas como negativas. La paradoja es lo Familiar-No familiar (5). Como ejemplo: cuando se recibe a una mujer que sufre violencia intrafamiliar y la/el enfermera/o le asiste en su proceso de transformación, brindando un acompañamiento y guía en la toma de decisión para que acepte recibir ayuda y rompa con el círculo de violencia, esto involucra trabajar con restablecimiento de autoestima, vínculos afectivos, relaciones interpersonales, género, espiritualidad y sexualidad.

En las situaciones de la vida diaria, cada principio con sus conceptos y paradojas actúan de manera simultánea, por lo tanto, para el profesional de Enfermería aplicar los principios significa respetar a las personas, sus valores y creencias, ya que ellos son, independientemente de sus habilidades personales, méritos, estatus social, facilidad de palabra, de su condición de salud o edad.

La Figura 2 ilustra los supuestos, postulados y principios con sus conceptos y paradojas que conforman la ontología de la Escuela del Pensamiento, los que se derivan de la Teoría Humanbecoming.

Figura 2: Humanbecoming supuestos, postulados, principios, conceptos y paradojas.

\begin{tabular}{|c|c|c|c|}
\hline Supuestos & Postulados & Principios & Conceptos y Paradojas \\
\hline $\begin{array}{l}\text { 1-Humanbecoming es estructurar } \\
\text { significados y tomar decisiones en } \\
\text { las situaciones de salud. }\end{array}$ & \multirow{4}{*}{$\begin{array}{l}\text { Infinito } \\
\text { Paradoja } \\
\text { Libertad } \\
\text { Misterio }\end{array}$} & $\begin{array}{l}\text { Estructurando } \\
\text { Significados }\end{array}$ & $\begin{array}{l}\text { Imagen } \\
\text { Valoración } \\
\text { Lenguaje }\end{array}$ \\
\hline $\begin{array}{l}\text { 2-Humanbecoming es configurar los } \\
\text { patrones rítmicos de relación con el } \\
\text { universo humano (humanuniverse). }\end{array}$ & & Configuración de & \multirow{2}{*}{$\begin{array}{l}\text { Revelación-ocultación } \\
\text { Permitir-limitar } \\
\text { Conexión-separación }\end{array}$} \\
\hline $\begin{array}{l}\text { 3-Humanbecoming es co- } \\
\text { trascender ilimitadamente con las } \\
\text { posibilidades que emergen. }\end{array}$ & & Patrones Rítmicos & \\
\hline $\begin{array}{l}\text { 4-Humanbecoming es co-crear en el } \\
\text { universo humano (humanuniverse) } \\
\text { una sinfonía perfecta. }\end{array}$ & & Co-trascendencia & $\begin{array}{c}\text { Poder } \\
\text { Origen } \\
\text { Transformación }\end{array}$ \\
\hline
\end{tabular}

Fuente: Traducido al español y modificado por las autoras. Parse, R. The Humanbecoming Modes of Inquiry (17). 


\section{Vivir el Humanbecoming}

La Escuela Humanbecoming es una opción de vida que eligen las/os enfermeras/as para llevar a cabo la práctica de la disciplina (6), sin embargo, una de las grandes limitaciones para difundirla ha sido el nivel filosófico del lenguaje que utiliza. Además por esta misma característica se dificulta la traducción de los términos a otros idiomas como el español y el portugués $(1,6,19)$. Sin embargo, las investigaciones presentadas demuestran que se realizan esfuerzos por difundir esta teoría en el idioma español y portugués, con el fin de poner a disposición de los/as enfermeros/as este conocimiento disciplinar y puedan decidir si desean profundizar en ella y aplicarla a las diferentes realidades profesionales.

La Escuela Humanbecoming reconoce que la/el profesional de Enfermería requiere desarrollar extensivamente conocimiento técnico y habilidades para proveer cuidado de forma segura y competente; sin embargo, Enfermería debe considerar que este conocimiento y habilidades necesarias no la definen, porque muchas de las acciones derivadas de las intervenciones médico-quirúrgicas no son ejecutadas exclusivamente por enfermeras/os, sino que también intervienen una serie de profesionales y técnicos de la salud $(5,31,32)$.

De este modo, la Escuela de Pensamiento Humanbecoming otorga herramientas a la/el Enfermera/o que le ayudan a definir su actuar en el ejercicio profesional, en cuanto logra responder preguntas y descubrir significados sobre las experiencias humanas de salud y calidad de vida de las personas usuarias. Esto le permite aprehender de las personas y de este modo unir su propio conocimiento con el obtenido de otras disciplinas y así dar a conocer a la sociedad cómo Enfermería desde su propio punto de vista aborda las experiencias humanas de salud y calidad de vida. Este conocimiento le brinda seguridad en su actuar, porque constituye un aporte disciplinar, que le permitirá interactuar de manera inter o transdisciplinaria con otros profesionales (33).

La práctica del Humanbecoming involucra la realización de un Proceso de Enfermería que fluya a partir del planeamiento e intervención conjunta entre la/el enfermera/o y la persona, de acuerdo con los valores que vive la enfermera/o en concordancia con la experiencia de las personas usuarias; incluye los significados, prioridades y preocupaciones que se identificaron como prioritarias (34), tiene como fin co-transformar sus patrones y significados en beneficio de su salud y calidad de vida. Es un Proceso de Enfermería fundamentado en el respeto a la persona usuaria, ya que las experiencias en salud no se pueden solucionar a menos que las personas lo decidan $(5,25)$.

Para este proceso se requiere estar con, esto es una experiencia relacional que se representa en la Teoría Humanbecoming, cuando a las palabras se les antepone el prefijo "co" (7). Se expresa en las relaciones enfermerapersona/familia/comunidad (5). Se considera dentro de este enfoque que este aspecto es lo que define al profesional de Enfermería. Se aplica en la práctica con la "verdadera presencia", entendida como "una especial manera de ser, con el cual la/el Enfermeralo está atenta/o al significado de los cambios que presenta la persona en cada momento, contribuye a la reorganización de sus valores prioritarios para la toma de decisiones a favor de su salud y calidad de vida" (35). Está focalizada en qué es importante para la persona en el aquí y el ahora (36). La/el enfermera/o está en verdadera presencia cuando co-existe con otros ("estar con"), tiene la sabiduría para afrontar las expresiones de significados y patrones de relación y conduce a las personas usuarias a explorar sus esperanzas, deseos y sueños, y este proceso de exploración inspira cambios en la calidad de vida y salud (36). El logro de este proceso se le conoce como una forma de evolución humana. 


\section{CONSIDERACIONES FINALES}

La Teoría Humanbecoming en su evolución se convirtió en una Escuela de Pensamiento, que se compone de supuestos, principios, conceptos, paradojas y postulados; que actúan de manera simultánea a la hora de interactuar con las personas y conforman la ontología y epistemología de esta Escuela, lo que hace posible llevar a la práctica la filosofía, a través de los modelos y metodologías específicas. De este modo, permite implementar el Sistema de Conocimiento en Enfermería Conceptual-Teórico-Empírico y brindar un servicio a la sociedad guiado por el conocimiento, para lo cual es necesario continuar con los esfuerzos de difundir la teoría en español, reduciendo las dificultades del lenguaje e idioma, con el fin de comprobar la utilidad de esta teoría en la práctica profesional y, de este modo, convertirla en una alternativa para las/os profesionales que deseen ejecutar la disciplina desde el $\mathrm{Hu}$ manbecoming.

El pensamiento Humanbecoming tiene como razón principal el ser humano en interrelación directa con el entorno, la calidad de vida y la salud. La Enfermería consigue definir su actuar en el ejercicio profesional, cuando logra responder preguntas y descubrir significados sobre las experiencias humanas de salud y calidad de vida de las personas usuarias, que es lo que se conoce como "estar con" y es una experiencia relacional que se representa en la teoría cuando a las palabras se les antepone "co".

\section{AGRADECIMIENTOS}

A Rosemarie Rizzo Parse, RN; PhD; FAAN; Pittsburgh, PA USA. Humanbecoming Theory. A Patricia S. Lyon, RN; MSc; Toronto, Ontario Canada, Secretary International Consortium of Parse Scholars (ICPS) y al
International Consortium of Parse Scholars (ICPS).

\section{REFERENCIAS}

1. Fawcett J. Contemporary Nursing Knowledge. Analysis and Evaluation of Nursing Models and Theories. 2ed. Philadelphia: F.A. Davis Company. 2005.

2. Parse R.R. The Human Becoming School of Thought: A Perspective for Nurses and Other Health Professionals. Sage Publications, Thousand Oaks. 1998.

3. Kerouac, Suzanne y otros. El pensamiento enfermero. España: Masson.1996.

4. Parse RR. Living Quality: A Humanbecoming Phenomenon. Nur Sc Q. 2013; 26(2): 111-15.

5. Pilkington B, Jonas-Simpson Ch. The Humanbecoming School of Thought: A Guide for Teaching-Learning. 2ed. Toronto: International Consortium of Parse Scholars. 2007.

6. Marriner A, Raile M. Modelos y teorías de enfermería. 7ed. Barcelona - España: Elsevier. 2007.

7. Parse R. Community: A Human Becoming Perspective. Massachusetts: Jones and Bartlett Publishers. 2003.

8. Thomas K, Riggs R, Stothart K. The Meaning of Changing Expectations. A Qualitative Descriptive Study. Nurs Sci Q. 2013; 26(1): 59-67.

9. Parse R.R. The humanbecoming school of thought in 2050. Nurs Sci Q. 2007; 20(4), 308-11.

10. Parse R. Hope: An International Human Becoming Perspective. Jones and Bartlett Publishers, Inc and National League for Nursing: U.S.A. 1999.

11. Parse R. New Humanbecoming Conceptualizations and the Humanbecoming Community Model: Expansions with Sciencing and Living the Art. Nurs Sci Q. 2012; 25(1): 44-52. 
12. Parse R. The humanbecoming LeadingFollow Model. Nurs Sci Q. 2008; 21:374.

13. Parse R. Qualitative Inquiry: The Path of Sciencing. USA: Jones and Barlett Publisher. 2001.

14. Maxwell S. Humanbecoming: Not just a Theory-It is a Way of Being. Nurs Sci Q. 2010; 23: 216-19.

15. Benzein E, Saveman B, Norberg A. The meaning of hope in healthy, nonreligious Swedes. West J Nurs Res. 2000; 22(3): 303-19.

16. Parse R. The human becoming theory: the was, is and will be. Nurs Sci Q. 1997; 10(1), 32-8.

17. Parse R. The Humanbecoming Modes of Inquiry Refinements. Nurs Sci Q. 2011; 24(1): 11-15.

18. Rodero-Sánchez V, Acebedo-Urdiales S, Vives-Relats C, Pérez-Gurrea C. Análisis de los posibles desde la teoría de Parse en una persona con Alzheimer. Index Enferm. 2006; 15(54): 44-47.

19. Silva FVF, Silva LF, Guedes MVC, Moreira TMM, Rabelo ACS, Ponte KMA. Cuidado de enfermagem fundamentado em Parse. Esc Anna Nery. 2013; 17(1): 11119.

20. Diccionario de la Real Academia Española 22ed [Internet]. Madrid: Real Academia Española; 2001. [citado 30 de noviembre 2012]. Disponible en: http:// lema.rae.es/drae/?val=paradoja.

21. Parse R.R. The human becoming theory: challenges in practice and research. Nurs Sci Q. 1996; 9(2), 55-60.

22. Mac Donald C, Jonas-Simpson Ch. Living with changing expectation for women with high-risk pregnancies. Nurs Sci Q. 2009; 27(1): 74-82.

23. Ribas DL, Borenstein MS, Padilha MICS. Iluminando as vivências de indivíduos em sofrimento psíquico de um caps em Florianópolis. Texto Contexto Enferm, Florianópolis, 2007 Jan-Mar; 16(1): 40-6.

24. Sant'Ana MM, Pereira VP, Borenstein MS, Silva AL. O significado de ser fami- liar cuidador do portador de Transtorno mental. Texto Contexto Enferm. 2011; 20(1): 50-8.

25. Gail M. Theorical Approaches in Nursing Practice: A Comparison of Roy and Parse. Nurs Sci Q. 1990; 3: 81-7.

26. Parse RR. Transforming healthcare with a unitary view of the human. Nursing Science Quarterly. 2002; 15(1): 46-50.

27. Parse R. Iluminations: The Human Becoming Theory in Practice and Research. Massachusetts: Jones and Bartlett Publishers. 1999. Capítulo 15 The Lived Experience of Suffering. Daly J. Pp 243-68.

28. Afrio J, Freitag LM. El convivir de la familia con un individuo con SIDA, ciego. Enfermería Integral [Internet] 1999 [citado 11 agosto 2013] 1 trimestre (48). Disponible en http://www.enfervalencia. org/pub/comunicacion/revista-ei.php

29. Morrow M. Feeling unsure: A universal lived experience. [Tesis Doctoral] Universidad de Loyola Chicago. 2006.

30. Parse RR. A human becoming perspective on quality of life. Nursing Science Quarterly. 2007; 20(3): 217.

31. Bauman S. Contrasting two Approaches in a Community-Based Nursing Practice with Older Adults: The Medical Model and Parse's Nursing Theory. Nurs Sci Q. 1997; 10: 124-130.

32. Baumann S. A Humanbecoming Program of Research. Nurs Sci Q. 2012; 25(1): 17-9.

33. Plummer M, Molzahh A. Quality of life in Contemporary Nursing Theory. A Concept Analysis. Nurs Sci Q. 2009; 22(2): 134-149.

34. Parse R. The human becoming school of thought: A perspective for nurses and other health professionals. Thousand Oaks, C.A.: SAGE Publications. 1998.

35. Schmidts S. A focus on Human Flourishing. Nurs Sci Q. 2010; 23(4): 290-95.

36. Parse R. The Humanbecomin Modes of Inquiry: Refinements. Nurs Sci Q. 2011; 24(1): 11-15. 